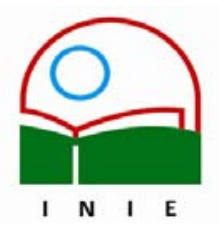

\title{
LA DIDÁCTICA DEL PROCESO DOCENTE PARA EL DESARROLLO DE LA PRÁCTICA LABORAL EN LAS EMPRESAS
}

\author{
Jorge Luis Herrera Fuentes ${ }^{1}$
}

Resumen: Como resultado de la investigación sobre el proceso docente-educativo por desarrollar en las unidades docentes, para la ejecución de la forma del proceso denominada práctica investigativo-laboral, se ha elaborado un modelo para la didáctica específica de este proceso, que por poseer características propias, ha sido necesaria la modelación de su propia didáctica, ésta es la propuesta que se presenta en el trabajo.

Palabras clave: PRÁCTICA INVESTIGATIVO- LABORAL/ UNIDADES DOCENTES/ DIDÁCTICA ESPECÍFICA/

Abstract: As a result of the investigation on the educational process to develop in the educational units, for the execution in the way of the process denominated investigative - labor practice; a model has been elaborated for the specific didactics of this process, that to possess characteristic very own it has been necessary to model her own didactics, this is the proposal that its presented in the work.

Key words: INVESTIGATIVE-LABOR PRACTICE / EDUCATIONAL UNITS / SPECIFIC DIDACTICS /

\section{Unidades docentes y práctica investigativo-laboral}

Desde la perspectiva conceptual, se ha seguido el criterio de considerar que la pedagogía y la didáctica son ciencias con objetos de estudio definidos y estrechamente interrelacionadas. Se asume que la didáctica es la ciencia social que estudia el proceso docente-educativo (su objeto) orientado a la formación de las personas. Hoy se debaten en la comunidad científica, los criterios de considerar a la didáctica como una ciencia o como una rama o disciplina particular de la pedagogía. Se ha asumido el primero, el de ser la ciencia del proceso docente-educativo. En este sentido, está estrechamente ligada con la pedagogía, cuyo objeto es el proceso de formación de las personas, ya que una parte decisiva de esta formación, transcurre en la escuela y de esta disciplina se ocupa la didáctica, que se ha ido independizando de la pedagogía, sin perder el vínculo que las une.

\footnotetext{
1 Doctor en Ciencias Pedagógicas, Universidad de Pinar del Río. Licenciado en Educación, especialidad de Física, en el Instituto Superior Pedagógico de Pinar del Río. Profesor Titular. Doctor en Pedagogía. Máster en Ciencias de la Educación. Vicedecano de la Facultad de Geología y Mecánica de la Universidad Hermanos Saíz Montes de Oca de Pinar del Río. Investiga en el campo de la Didáctica de la Educación Superior, en particular en la formación de las habilidades necesarias para los modos de actuación de los futuros profesionales y su capacitación laboral en las unidades docentes. Correo electrónico: herrera@geo.upr.edu.cu
}

Artículo recibido: 15 de junio, 2004

Aprobado: 8 de noviembre, 2004 
El proceso docente-educativo que se desarrolla en las unidades docentes para el desarrollo de la práctica investigativo-laboral, tiene especificidades que lo hacen poseedor de sus propias relaciones dialécticas y regularidades propias. Este tipo de proceso docenteeducativo resulta sui generis y, por supuesto, contiene su propio análisis didáctico, por eso para él se ha estructurado una didáctica específica.

La práctica investigativo-laboral es la forma organizativa de participación activa de los estudiantes, docentes y profesionales de la producción y los servicios, al interactuar entre sí y la comunidad (sociedad) durante la solución de los problemas reales que requieren el uso del método científico-investigativo propio del modo de actuación profesional, motivando el trabajo multidisciplinar y en equipos, y desarrollando la independencia y creatividad. Es la forma fundamental de la ley "relación universidad-sociedad" y es común a los procesos universitarios fundamentales: docencia, investigación y extensión... (Lazo, 1996, p. 68).

Las unidades docentes son empresas vinculadas a la universidad, que por sus condiciones objetivas y subjetivas, permiten el trabajo conjunto en el desarrollo de los componentes curriculares: académico, laboral-productivo, investigativo y de autopreparación, y de los procesos universitarios de docencia, investigación, extensión, y gestión, que aseguren la formación del profesional de perfil amplio desde el pregrado, el adiestramiento y la especialización, de una o varias carreras, mediante la solución de problemas reales técnico-profesionales de la producción o los servicios. (Herrera, 2003, p. 91).

El trabajo presenta una didáctica específica para el proceso docente-educativo en las unidades docentes para el desarrollo de la forma denominada práctica investigativo-laboral, en la cual se manifiestan las regularidades del proceso.

No se ha denominado didáctica especial por estar reservado este término para aquellas referidas a la enseñanza de disciplinas científicas (física, química, matemática, historia, entre otras), ni particular, por estar este término reservado para aquellas que estudian el proceso docente-educativo en condiciones o situaciones específicas dadas por el tipo de alumno (didáctica de la educación especial); o el nivel de escolaridad (didáctica de la educación preescolar, primaria, secundaria, universitaria). Parte de una didáctica particular, la universitaria, pero tiene su especificidad para el tipo de proceso. Se ha denominado específica, porque al investigar el tipo de proceso docente-educativo que se da en una empresa, con relaciones esenciales y regularidades propias, es necesario identificar cada 
categoría de la didáctica, con el proceso docente-educativo específico y esto es una prerrogativa de la didáctica especifica para este proceso, es un análisis didáctico del mismo. Partiendo del concepto de que la didáctica es una ciencia independiente, se necesita estructurar, sobre bases científicas y teóricas, la gestión de un proceso docente-educativo que actualmente se apoya en presupuestos esencialmente empíricos. El proceso formativo en lo investigativo-laboral que se desarrolla en las unidades docentes, se diferencia del proceso docente que se ejecuta en el campus de la universidad en que, el docente y los estudiantes conocen de antemano el comportamiento de todos o casi todos los componentes del proceso: objetivos, contenidos, métodos, medios, forma.

Si la práctica investigativo-laboral que desarrolla el estudiante, parte de la concepción teórica del aprendizaje, sobre la base de la solución científica de un problema profesional-laboral real en la empresa, entonces se comprenderá que el contenido de los componentes del proceso docente-educativo cambiarán en función de adecuarse al problema real que existe en dicha empresa.

La gestión del proceso juega un importante papel, porque las y los sujetos involucrados en él, son los que determinan el comportamiento de cada componente del proceso. Al impartir una clase, un tema o todo un programa, en el aula, el docente conoce de antemano el contenido de todos los componentes del proceso y el estudiante asimila este conocimiento de forma sistemática, generalmente conoce inclusive los próximos contenidos. Aquí la simulación de los procesos y los métodos reproductivos tienen un papel preponderante. Al llegar a la empresa, el que enseña no conoce nada o casi nada del comportamiento del proceso a desarrollar, todo parte del conocimiento del o los problemas por enfrentar; algo similar ocurrirá después con el estudiante. Hay que construir el proceso y en ello la gestión juega el papel principal. Aquí la modelación y los métodos creativos son los primordiales.

\section{Didáctica del proceso-educativo en la unidad docente}

\subsection{La didáctica como ciencia}

La pedagogía derivada del enfoque histórico-cultural en la formación de las personas, y ha hecho precisiones en la epistemología de la didáctica. Su concepción ha evolucionado desde ser considerada una rama o disciplina de la pedagogía, hasta una ciencia independiente. La generalidad de los autores contemporáneos la consideran como una rama o disciplina particular de la pedagogía, pero con independencia propia, al tener precisado su objeto de estudio. Coinciden en que es una teoría general de la instrucción, la enseñanza e inclusive la educación; al considerar que los procesos de instrucción y enseñanza están 
indisolublemente ligados. En la medida en que han avanzado las investigaciones científicas en el campo de la didáctica, ésta se ha ido independizando como ciencia, al precisarse sus métodos propios y leyes generales y particulares.

Danilov y Stkatkin (1978, p. 28) la consideran una disciplina científica independiente; Labarrere y Valdivia (1989, p. 11), como una ciencia independiente, aunque la definición del objeto de estudio queda reducido a la instrucción (ligado al proceso educativo) o al tipo de enseñanza y aprendizaje que se da en la escuela, limitada ésta a un tipo de institución algo cerrada.

Álvarez de Zayas (Álvarez de Zayas, C. y González, E.U. 1998, p. 5) la define como la ciencia del proceso docente-educativo (objeto de estudio) que se establece en una institución educativa y con la orientación de los docentes. Este concepto de didáctica se corresponde con las concepciones de este trabajo, si se precisan los términos de "institución educativa" y "docentes", y van más allá de la escuela y los profesores.

¿Qué características se destacan en este proceso docente- educativo?

- El contenido de aprendizaje es diferenciado para cada estudiante y fundamentalmente extracurricular, responde a las necesidades derivadas de la búsqueda del conocimiento para la solución de un problema de investigación.

- El estudiante logra la apropiación del contenido de forma autorregulada, por medio de su trabajo investigativo; la fuente del mismo está en la práctica social.

- El método de trabajo se fundamenta en la unión de lo investigativo - productivo.

- El estudiante asume una responsabilidad social en el proceso de aprendizaje, al existir una expectativa en el impacto de su resultado investigativo.

- Los sujetos que intervienen construyen el proceso a partir de un problema laboral real del perfil profesional; cada componente del mismo se particulariza en un aprendizaje individualizado, de trabajo colectivo y multidisciplinar.

\subsection{Didáctica universitaria}

En la pasada década, culminó un proceso de conformación de una epistemología para la didáctica de la educación superior en Cuba, dando continuidad a la estratificación de esta ciencia por niveles de enseñanza y en la que ya existen resultados consolidados en los niveles primario y medio.

Álvarez de Zayas (1995, p.12) fue el pionero en establecer una didáctica para la educación superior, basado en su Teoría de los Procesos Conscientes, que a su vez, se fundamenta en 
la Teoría de la Concepción de los Procesos Sociales de González (1993, p. 36) y con base filosófica en el materialismo dialéctico e histórico.

Partiendo de este mismo marco teórico, se han elaborado otros modelos de didáctica para la educación superior, como el Holístico Configuracional de Fuentes (1998, p. 33; 2000, p. 64) y el elaborado por Vargas (s.f., pp. 43-64), así como los de González y col. (1995, p. 3); Addine (1997, p. 55; 2002, p. 1); Addine y otros (2002, p. 8); Salazar (2001, p. 49). Estos modelos han enriquecido la epistemología de la didáctica de la educación superior y han aparecido definiciones de nuevas categorías didácticas.

A continuación aparece una breve discusión de las categorías que se incluirán en la propuesta que se presenta en el trabajo:

Problema: La concepción contemporánea de la metodología de la investigación científica, reconoce de modo casi universal, la importancia de comenzar por el planteamiento del problema como génesis de toda investigación científica. La didáctica es una ciencia en desarrollo y el problema debe aparecer como un componente primario de su concepción teórica, de modo que mantenga en estado latente la necesidad permanente del perfeccionamiento del proceso que estudia. Autores como Danilov y Stkatkin (1978, p. 34); Álvarez de Zayas (1999); Castellanos y otros (2001), incluyen el problema como componente de sus concepciones didácticas, aunque debe reconocerse que resulta el componente más polémico.

En todos los casos, el problema se relaciona con la necesidad de la sociedad de preparar a sus ciudadanos para el desarrollo material y cultural, de lo que se derivan tareas para la escuela, que es quien responde a esa necesidad social.

Objeto de estudio: Definir el objeto de estudio de la didáctica es plantear su misión como ciencia. En esto coincide la generalidad de los autores, aún cuando lo denominan de modo diferente: proceso de enseñanza - aprendizaje en la escuela (Klingberg, 1990, p. 32; González y col. 1995; Addine, 1997, 1998, 2002; Addine y otros, 2002; Colectivo de autores del IPLAC, 1999; Salazar, 2001); de instrucción y enseñanza ligado a la educación (Danilov y Skatkin, 1979, p. 11; Tomaschewski, 1966, p. 25; Babanski, 1982, p. 18; MINED, 1984; Castellanos y otros, 2001; Silvestre y Zilberstein, 2000), o docente - educativo (Álvarez de Zayas, 1999; Vargas, s/f ; Fuentes, 2000; Colectivo de autores del CEPES, 1995)

Objetivo: La mayoría de las concepciones didácticas de diferentes autores, plantean de modo más o menos claro su objetivo; así Danilov y Stkatkin lo plantean como problema crucial de la didáctica (Danilov y Stkatkin, 1981, p. 27); Klingberg lo define como tarea (Klingberg, 1990, p. 37); Álvarez de Zayas lo refiere como tarea crucial de la didáctica 
(Álvarez de Zayas y González, 1998; p. 7); Vargas (s/f) llega a plantear, en forma de tareas, hasta seis.

Leyes: Diversos autores incluyen conjuntos de leyes denominados de esta forma o con otros términos. Así por ejemplo: Danilov y Stkatkin (1981, p. 29) definen tres leyes generales de la enseñanza; en MINED (1984, p. 30) se definen cinco leyes del proceso de enseñanza; Klingberg (1990, p. 223) define ocho leyes de la didáctica o relaciones didácticas legítimas; Álvarez de Zayas (1990) define cuatro leyes en su libro Pedagogía, el mismo autor (1995) las reformula es dos propuestas y el propio autor (1998) las plantea con otra formulación; Vargas (s.f.) plantea dos leyes y Fuentes (2000, p. 83; 1998, p. 44) define dos. Las dos leyes de la didáctica, en la forma expresada por Álvarez de Zayas, sintetizan, a juicio de este autor, la dinámica de las relaciones esenciales entre los componentes del proceso, se han expresado en el lenguaje de lo investigativo-laboral, de forma que respondan a la didáctica específica en la unidad docente.

Principios: Los diferentes modelos de la didáctica derivados del enfoque histórico - cultural han incluido sistemas de principios didácticos. Estos conjuntos de principios muestran los lógicos cambios derivados de la necesidad de adaptar el proceso docente - educativo a las exigencias que impone la sociedad a la escuela en cada época histórica. Se conocen principios didácticos desde las "reglas de enseñar" elaboradas por Comenio (1983, p. 114), hasta los sistemas de principios propuestos por distintos pedagogos seguidores del enfoque histórico - cultural.

Los principios son las reglas o postulados, de mayor nivel de generalidad, que abarcan a los objetos y fenómenos de una parte de la realidad objetiva que se estudia y de la que se han hecho abstracciones y generalizaciones. Etimológicamente, se deriva del latín principium que significa punto de partida, regla principal, idea rectora.

En la pedagogía y didáctica cubanas se establecieron también un sistema de nueve principios didácticos (Labarrere y Valdivia, 1988; p. 56) centrados en establecer normas generales de enseñanza; es decir, su centro de atención abarca fundamentalmente la actividad del que enseña.

En la forma breve y concisa en que llegaban a los docentes para normalizar el proceso docente - educativo (educativo, científico, asequible, sistémico, teórico - práctico, consciente y activo, diferenciado, y audiovisual), muchas veces se tomaba su cumplimiento como criterio único de calidad del proceso, marcando una tendencia a la esquematización y uniendo la riqueza y creatividad del mismo. El otro sujeto del proceso (el que aprende) quedaba fuera de la valoración. Un sistema de principios didácticos, en su condición de reglas más 
generales del proceso docente-educativo, debe ser abarcador de todas las dimensiones y componentes del mismo y, a su vez, requiere tener la suficiente flexibilidad que les permita determinar estos componentes independientemente de la tipología que se utilice para clasificarlos; en ello reside su función transformadora.

\section{Didáctica del proceso docente - educativo en la unidad docente}

Después de analizados numerosos (más de veinte) modelos de didáctica en sus componentes y estructura, se decidió proponer el que se esquematiza en la figura 1 y en el que se manifiestan las regularidades de este proceso docente - educativo.

\subsection{Regularidades de la didáctica del proceso docente - educativo para la formación investigativo - laboral en las unidades docentes:}

1. Integra y define las categorías didácticas en las que se manifiestan las relaciones presentes en el objeto de estudio, con sus contradicciones dialécticas:

Leyes

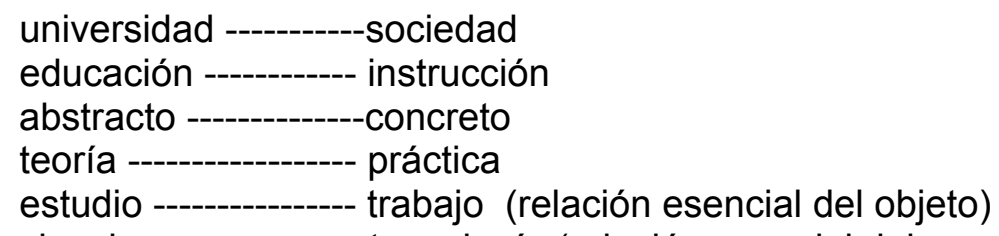

2. Las categorías didácticas: problema y educación tienen el carácter principal en el proceso docente - educativo que se gestiona por medio de esta didáctica.

3. Fundamenta la calidad del proceso didáctico en la calidad de la gestión que del mismo hacen los sujetos involucrados y en la calidad de la relación universidad-empresa, para la cual se define un modelo.

4. Concibe al proceso como una etapa en el desarrollo de las competencias profesionales, el cual se enlaza en un nexo ascendente hasta el postgrado, por lo que lo fundamental es capacitar a los sujetos en el autoaprendizaje.

5. Utiliza un marco teórico basado en la filosofía marxista-leninista, su teoría del conocimiento y en la didáctica derivada del enfoque histórico-cultural 
6. Define la integración interdisciplinar de objetivos, contenidos y métodos en la investigación que da solución al problema (multidisciplinar) de la empresa.

7. Concibe a los sujetos participantes del proceso didáctico como autores co-gestores de la enseñanza y el aprendizaje en el marco de la unidad docente.

8. Concibe a la empresa - unidad docente como el sistema de medios para el desarrollo del proceso didáctico y, en consonancia con ello, lo modela.

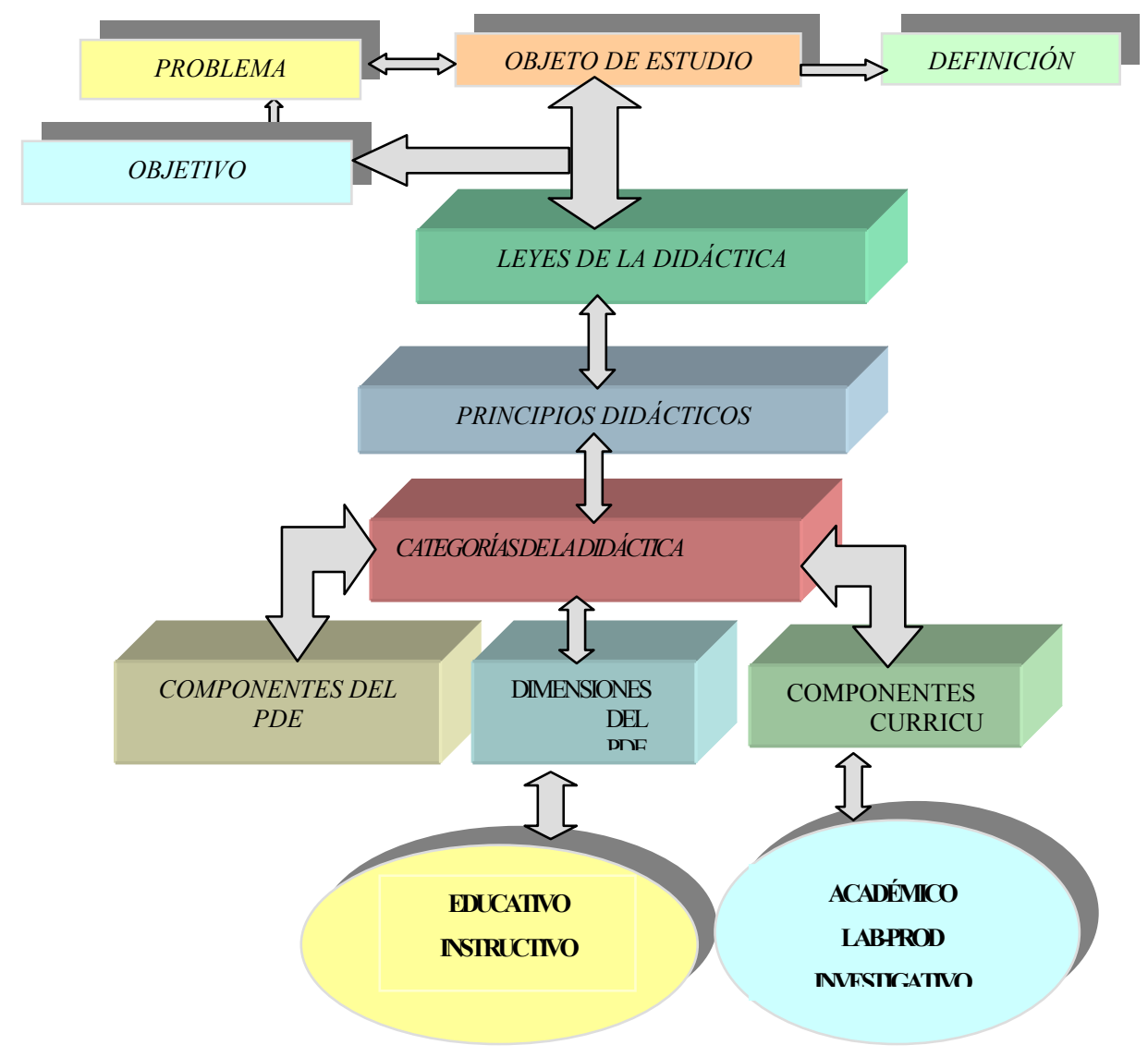

Figura 1: Didáctica del proceso docente - educativo en la unidad docente

\subsection{Descripción de los componentes de la didáctica}

3.2.1 Problema: Necesidad de lograr una formación investigativo- laboral eficaz y eficiente en los estudiantes durante su tránsito por las unidades docentes.

3.2.2 Objeto de estudio: El proceso docente-educativo dirigido a la formación investigativo- laboral de los estudiantes universitarios, la cual se desarrolla en las unidades docentes. 
3.2.3 Objetivo: Estructurar las interacciones más apropiadas entre las categorías de la didáctica (dimensiones, componentes, principios, leyes y regularidades del proceso docenteeducativo) para lograr un proceso de formación investigativo- laboral eficaz y eficiente en las unidades docentes.

3.2.4 Definición: La didáctica del proceso docente-educativo en las Unidades Docentes estudia las particularidades del proceso formativo que se produce en las condiciones específicas de estas, durante el desarrollo de la práctica investigativo - laboral.

\subsubsection{Leyes de la didáctica}

a) Dinámica de las relaciones entre los componentes problema - objeto - objetivo: La calidad del proceso docente-educativo para la formación investigativo- laboral que se desarrolla en las unidades docentes; la determina el nivel de desarrollo científico - cultural de la sociedad, que se manifiesta en la empresa vinculada con la universidad donde se insertan los estudiantes, y presupone una clara definición de un problema profesional que debe resolver el estudiante en la empresa, que existe en un objeto bien determinado y que define un claro objetivo de la actividad por desarrollar. Esta ley presenta la dinámica de la relación entre los componentes problema - objeto - objetivo. El problema se da en el objeto como una necesidad presente en la sociedad de resolver una situación que requiere ser modificada. El problema tiene el carácter rector para la empresa.

El objetivo, como estado futuro deseado del objeto, es una visión del sujeto (carácter subjetivo) que, como proyección se formula en el presente, pero como resultado tangible (carácter objetivo) se obtendrá solo cuando se solucione el problema. De esta manera, el objetivo se convierte en el componente rector del proceso para la universidad.

b) Dinámica de las relaciones entre los componentes objetivo-contenido-método: La calidad de la formación investigativo-laboral de los estudiantes universitarios en la unidad docente, es determinada por la capacidad de instruir mediante la educación en el proceso, lo que se manifiesta en la clara definición del contenido, los métodos de aprendizaje y los modos de actuación profesional que determinan los objetivos de la actividad del estudiante. Esta ley dinamiza la unidad del contenido y el método con el objetivo.

El objetivo determina el contenido y la estructura que este asume en el proceso, el contenido es función del objetivo. La relación objetivo-contenido-método determina la dinámica esencial del proceso. El método es la vía, el modo de desarrollar este proceso, guía el proceso de asimilación del contenido para alcanzar el objetivo. En la unidad docente, el 
objetivo determina que el método en lo general tienda en los niveles de asimilación de creación-innovación con un alto grado de independencia cognoscitiva.

En el contexto de la unidad docente, el vínculo contenido-método expresa la relación entre el sujeto con su objeto de estudio; esto se aprecia en el componente laboral, pero también el sujeto, convertido en investigador, actúa sobre el objeto real como parte importante de su formación en el proceso docente-educativo. Esto da una connotación relevante al método de investigación. El sujeto de aprendizaje alcanza en la unidad docente un grado predeterminado de formación profesional investigativo- laboral, al tiempo que logra, formular propuestas o enfoques novedosos (invención) a la solución de problemas de la producción o los servicios (innovación). Esta relación que se amplía de esta forma al objetivo y al problema, denota el carácter preponderante de la actividad investigativo- laboral en la unidad docente. Por ello los métodos en la unidad docente deben caracterizarse por una orientación hacia los de carácter de innovación-creación, con base en lo problémico, que propendan en niveles de asimilación innovativos-creativos.

\subsubsection{Principios didácticos del proceso docente-educativo en la unidad docente}

Como los principios didácticos deben responder a la realidad socio-histórica y a los avances científicos que han permitido profundizar en las concepciones de la pedagogía y la didáctica, se propone un sistema que se identifique con el proceso de formación investigativo- laboral que se desarrolla en las unidades docentes.

El punto de partida del sistema de principios didácticos que se propone, es la propuesta de Silvestre (Silvestre y Zilberstein, 2000, p. 22) y se han identificado con el tipo de proceso docente-educativo que trata el trabajo. La propuesta recoge las concepciones clásicas, logrando una cualidad superior y poniéndolos en función del que aprende, además, se identifican con el tipo de proceso específico del campo de acción, para su mejor comprensión, sin que con ello pierdan su nivel de generalidad para el desarrollo del proceso en otros contextos, de modo que quedan así:

- Principio del diagnóstico de las potencialidades y nivel de partida de los contenidos del que aprende, para enfrentar la práctica investigativo-laboral en la unidad docente.

- Principio de la motivación hacia el objeto de estudio, a través del desarrollo de la necesidad de aprendizaje que propicia la apropiación de los conocimientos necesarios para buscar solución a un problema profesional laboral real, usando el método de la investigación científica. 
- Principio de la capacitación para la solución de problemas profesionales reales en su esfera de actuación.

- Principio de la activación del que aprende para la búsqueda, exploración y reflexión del contenido necesario para su desarrollo social - profesional, en el contexto de la sociedad cubana contemporánea.

- Principio de la vinculación del contenido con la ejecución de los procesos universitarios que se desarrollan en la unidad docente, durante la práctica investigativo- laboral.

- Principio de la atención a las diferencias individuales de los que aprenden.

- Principio de la interacción del trabajo individual del que aprende con el resultado colectivo grupal multidisciplinar en la solución de los problemas profesionales laborales de la empresa.

- Principio del desarrollo de la capacidad del autoaprendizaje en los que aprenden.

\subsubsection{Las dimensiones del proceso docente-educativo en la unidad docente}

La relación de lo educativo-instructivo-desarrollador está presente en el proceso; lo educativo adquiere el papel principal, a la vez, que transforma su personalidad (se educa) el estudiante asimila nuevas competencias profesionales. Asimila nuevos contenidos; amplía su currículo (se instruye) y, en la dinámica de su trabajo creativo, transforma el medio y a sí mismo (se desarrolla).

\subsubsection{Unidad educación-instrucción.}

Todo proceso docente-educativo lleva en sí mismo, la unidad de lo instructivo y lo educativo, no importa el nivel; podrán cambiar los métodos y las formas, pero ningún proceso de formación puede ser exclusivamente instructivo. Por tanto, esta unidad tiene que caracterizar también al desarrollado en la unidad docente.

La relación entre la educación y la instrucción, en general, plantea el logro de la instrucción a través de la educación, dado que durante la ejecución de la práctica investigativo-laboral en la empresa, lo más trascendental en el aprendizaje del estudiante, son los factores educativos, como es la apropiación de los valores, la ética, la disciplina (laboral y tecnológica) del trabajo. Estos son también contenidos que se integran al currículo ampliado del estudiante, que los capacitan para operar con los contenidos del perfil profesional en función de cumplir con los objetivos de la sociedad y, por ello, devienen en competencias profesionales. 


\subsubsection{Los componentes curriculares del proceso educativo en la unidad docente}

El proceso permite la sistematización y ampliación del currículo académico del estudiante, por medio de su trabajo investigativo en la búsqueda de soluciones a un problema laboral real, en lo cual la autopreparación, que le permite aprovechar la cultura laboral de la empresa, sus medios y el entorno social de la misma, se convierte en un factor dinamizador del resto de los componentes y lo pone en condiciones de continuar aprendiendo en otros contextos, aún después de graduado.

\section{Componentes del proceso docente - educativo en la unidad docente}

A continuación se incluye una breve discusión sobre los componentes del proceso, sintetizados en la figura 2 .

\subsection{Caracterización del proceso docente - educativo en la unidad docente}

El proceso docente - educativo en la unidad docente, tiene en sí mismo todas las características y rasgos generales que fueron definidos para estos procesos. Además tiene sus especificidades esenciales que marcan sus diferencias, permitiendo identificarlo como una forma particular de la formación investigativo-laboral.

En la unidad docente, la actividad investigativo- laboral tiene un papel preponderante, llegando a constituir el eje central del proceso. El estudiante va asimilando e integrando nuevos contenidos de una forma esencialmente diferente de la de otros procesos formativos, en la relación que establece el sujeto que aprende con su objeto de estudio, durante la investigación que transcurre en el proceso laboral, consolidando el carácter científico del mismo. Desde esta óptica, los métodos y la apropiación de los contenidos son de una naturaleza y dinámica diferentes, con una participación más activa y protagónica del sujeto del aprendizaje convertido al propio tiempo en investigador - trabajador, donde la lógica de la ciencia es el método de aprendizaje. La intención de la unidad docente es formativa, el proceso docente - educativo que se desarrolla principalmente mediante la actividad laboral y la investigación científica, resolviendo problemas laborales de la empresa, con el objetivo de formar profesionales de elevadas competencias y valores. Asimismo, se diferencia de los procesos investigativos puros, del proceso laboral puro objeto de la profesión y también de otros procesos docente - educativos de pregrado.

\subsection{Definición de proceso docente-educativo en la unidad docente: Es el} proceso que procede de las relaciones que se establecen entre los sujetos que lo gestionan 
(los que enseñan y los que aprenden), y se dirige de un modo sistémico, eficaz y eficiente, a la formación investigativo-laboral de los estudiantes universitarios, en las dimensiones educativa, desarrolladora e instructiva (objetivo), para dar solución a la necesidad social de lograr una formación con calidad en los graduados (problema); mediante la apropiación de la cultura de trabajo de un centro laboral y su entorno (contenido), en el que debe enfrentar un problema profesional laboral real cuya solución encontrará por medio de la investigación científica (método); en el plazo establecido para el desarrollo de la práctica investigativo laboral (forma); en la empresa constituida en unidad docente de la universidad (medio); y que debe generar un resultado científico listo para su introducción en la práctica, que determina la calidad del proceso (evaluación); y cuya dinámica está determinada por las relaciones causales entre esos componentes y de ellos con la empresa y su entorno social (leyes), que constituyen su esencia (Identificado para este proceso, de la definición de Álvarez de Zayas, 1999).

\subsection{Las ideas rectoras del proceso docente - educativo en la unidad docente}

- Formar lo educativo en el trabajo: El estudiante en la universidad, y en particular, en la unidad docente tiene que formarse en el trabajo.

- Utilizar el método investigativo-productivo en la solución de problemas: El diagnóstico y la solución de los problemas de las empresas, que son los elementos más dinámicos, tienen que hacerse con los métodos de la ciencia, en este proceso la modelación de los procesos es fundamental.

\subsection{Características del proceso docente-educativo en la unidad docente}

a) Motivador: Planteador de necesidades de aprendizaje.

b) Problematizador: Planteador de nuevos problemas.

c) Concientizador: Formador de la conciencia del trabajo

d) Polémico, discusivo.

e) Orientador hacia la meta u objetivo.

f) Provocador de tensiones y conflictos que contribuyan a estimular el desarrollo personal y no de resistencias al aprendizaje.

g) Diferenciado: respetador de las características individuales del estudiante.

h) Centrado en el estudiante.

i) Integrador: De lo cognoscitivo, lo afectivo, lo axiológico.

j) Vocacional-profesional. 
k) Socio - educativo: Desarrollador de la colaboración, cooperación e interdependencia positiva.

I) Más que enseñar el ejercicio de la profesión, debe lograr un estudiante capaz de aprender en otros contextos laborales.

m) Debe contribuir a que el estudiante:

- Aprenda a conducirse en diferentes medios sociales

- Asimile valores y normas sociales propias del centro laboral y la comunidad.

- Realice la disciplina del trabajo (horario, asistencia, puntualidad, normas).

- Se encauce hacia su futuro empleo.

- Se forme técnico - profesionalmente.

- Logre el trabajo de los estudiantes sobre la búsqueda de la solución de problemas laborales reales (trabajo en ambiente real).

n) Debe propiciar el contacto del estudiante con nuevas tecnologías y contenidos no ofrecidos por la universidad en la educación formal

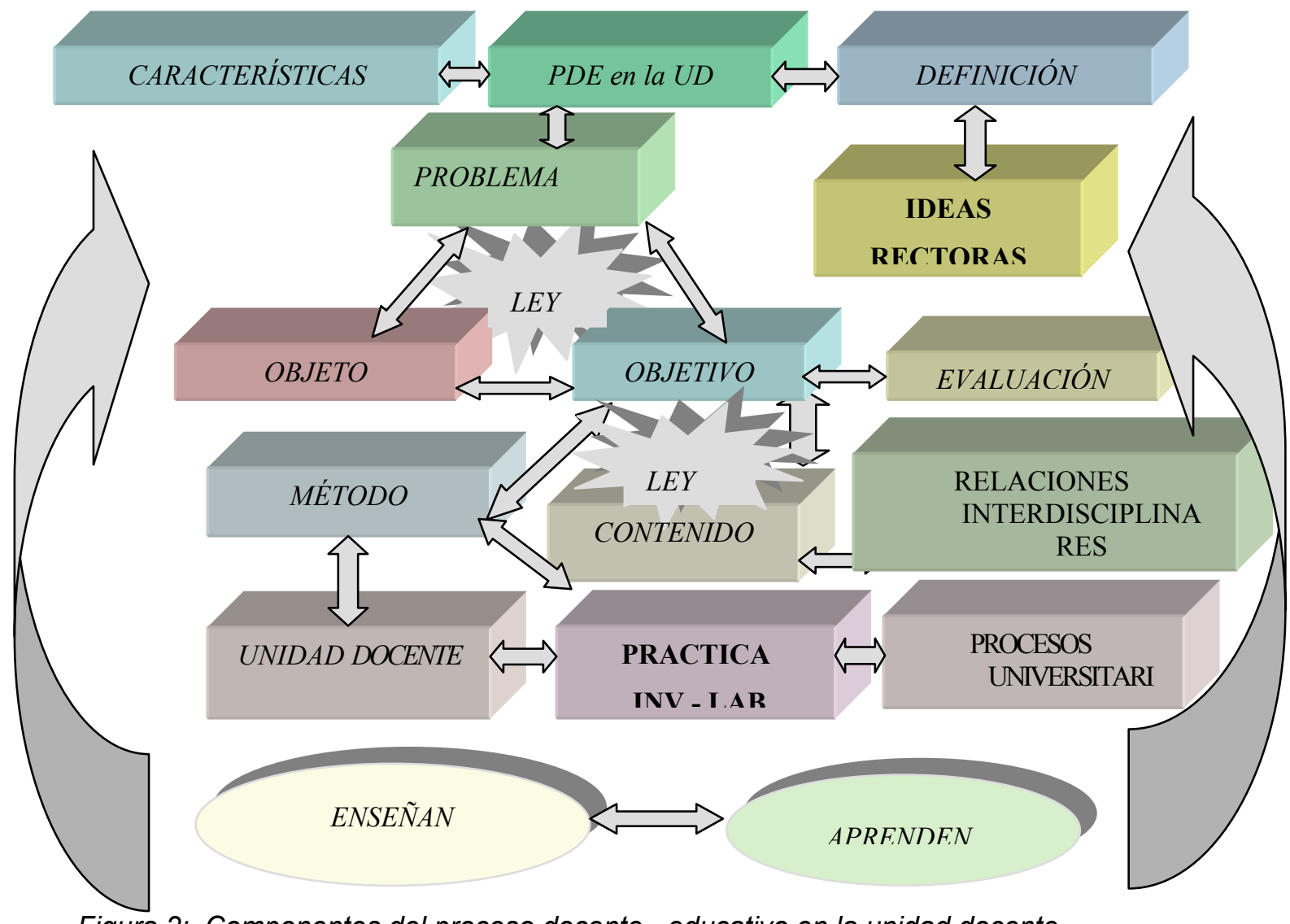

Figura 2: Componentes del proceso docente - educativo en la unidad docente 
o) Debe formar la ética laboral del estudiante.

p) Formador de hábitos profesionales.

q) Socializador: Formador de las personas a través de las relaciones sociales entre los estudiantes, los profesores de la universidad, los sujetos de la empresa y la comunidad del entorno social.

r) No represivo.

s) Sistematizador: Reforzador de lo ya aprendido.

t) Dirigido: Planificado, organizado, ejecutado en el tiempo y el espacio, controlado.

u) Innovativo - creativo.

\subsection{Los sujetos actuantes del proceso docente - educativo en la unidad docente}

Los sujetos del proceso son: Los que enseñan, dentro de los que se incluyen los profesores, profesionales, técnicos y trabajadores de la empresa capacitados para la enseñanza, y los que aprenden, los estudiantes insertados.

\subsubsection{Cualidades del sujeto que enseña en la unidad docente:}

- Estimuladores de la actividad de las y los estudiantes.

- Con visión optimista del que aprende.

- Facilitadores y orientadores del aprendizaje.

- Empáticos, comprensivos.

- Auténticos (se asumen a sí mismo).

- Aceptadores de los demás.

- Motivadores.

- Desarrolladores de la autoestima de los que aprenden.

- Afectivos.

- Preparado en su ciencia y que posea un perfil amplio en la misma.

- Actualizado.

- Ser un investigador, dar el ejemplo de lo que se pretende del estudiante.

- Poseer una cultura general integral.

- Sólida formación psicopedagógica.

- Un educador ejemplo, honrado, honesto, consagrado, ético en su profesión y con buenas relaciones humanas.

- Facilitador del aprendizaje. 
4.5.2 Cualidades del sujeto que aprende en la unidad docente:

- Activos.

- Autónomos, responsables de su actuación, independientes, capaces de decidir lo que desea con su actuación y de lograrlo.

- Auto reflexivos, autorregulados en su conducta.

- Participantes activos de su aprendizaje.

- Creadores.

- Debatidores, polémicos.

- Aceptadores de los demás y de sí mismos.

- Asumidores de las metas propuestas.

- Motivados.

- De elevada autoestima.

\subsection{El problema}

Se asume como definición, la situación que presenta un objeto y que genera una necesidad social. El encargo social es un problema, porque en éste se concreta la necesidad social de preparar a los ciudadanos con determinada formación, conocimientos, habilidades y valores, para actuar en el contexto propio de dicha sociedad. La necesidad social de que los hombres que resuelven problemas laborales de la producción y los servicios tengan un alto nivel de preparación científica y laboral, para lograr resultados de calidad, con eficiencia y eficacia, es el problema de este proceso.

\subsection{El objeto}

El objeto es aquel componente del proceso docente - educativo que expresa la configuración que este adopta como portador del problema y que en su desarrollo lo transforma, dándole solución y alcanzando el objetivo. Es la parte de la realidad en la que existe el problema, es un aspecto del proceso productivo o de servicio, en el cual se manifiesta la necesidad de capacitar a los futuros profesionales para que participen en la solución del problema.

\subsection{El objetivo}

Es la aspiración que se pretende lograr en la formación de los estudiantes, para resolver el problema. El objetivo es la aspiración, el propósito, que se quiere formar en los estudiantes: 
la instrucción, el desarrollo y la educación. Es la categoría rectora del proceso docente educativo, en el contexto de la universidad.

El objetivo del proceso docente - educativo en la unidad docente es el de capacitar al futuro profesional en la solución de los problemas reales de la producción o los servicios en los centros laborales de su campo de actuación, utilizando para ello el método científico. El objetivo a desarrollar en el contexto de las unidades docentes en lo educativo, académico, investigativo y laboral puede sintetizarse en: contribuir a la formación de un egresado con altos valores morales, éticos, estéticos y profesionales, con amplio grado de independencia y competencia, capaz de enfrentar múltiples situaciones y transformarlas en un marco de sustentabilidad, en el contexto de una sociedad.

\subsection{El contenido}

Para alcanzar el objetivo, el estudiante debe formar su pensamiento y cultivar sus facultades, mediante el dominio de una rama del saber, de una ciencia, de parte de ella o de varias interrelacionadas entre sí y que expliquen el comportamiento del objeto en que se manifiesta el problema "es aquella parte de la cultura y experiencia social que debe ser adquirida por los estudiantes y se encuentra en dependencia de los objetivos propuesto" (Addine, 1998, p. 22); "es el conjunto de valores materiales y espirituales creados por la humanidad en el proceso de la práctica histórico - social y caracteriza el nivel alcanzado por la sociedad" (Álvarez de Zayas, R: M; 1997, p. 34).

El contenido de la enseñanza - aprendizaje está integrado de forma unitaria por el sistema de conocimientos, el sistema de habilidades, el sistema de valoraciones, normas de actuación y el sistema de experiencias de la actividad creadora acumulados por la humanidad en el desarrollo histórico - social del proceso educacional como fenómeno social y sus resultados (Danilov y Skatkin, 1981, p. 195). El contenido es el componente del proceso docente - educativo que expresa aquellos aspectos necesarios e imprescindibles para cumplimentar el objetivo y que se manifiesta en la selección de los elementos de la cultura que debe aprender el estudiante, para alcanzar los objetivos.

Estos son los contenidos que se enseñan y se aprenden.

- Sistema de conocimientos.

- Sistema de habilidades y hábitos.

- Sistema de relaciones con el mundo.

- Sistema de experiencias de la actividad creadora... 
(A. M. González, S. Recarey, F. Addine, en Addine, F. y otros, 2002, p.72)

En el contenido se distinguen tres dimensiones: los conocimientos, que reflejan el objeto de estudio; las habilidades, que recogen el modo en que se relaciona el hombre con dicho objeto; y los valores, que expresan la significación que el hombre le asigna a dichos objetos. Hoy se incluye la creatividad como una cuarta dimensión, que adquiere un papel relevante en el proceso que se da en la unidad docente, que se encamina a buscar una solución real a un problema también real.

En la creatividad se expresa la esencia sociotransformadora del hombre, ... como categoría fija el aspecto de la actividad humana que se orienta al futuro ... como resultado de la cual se forma un nivel más alto del conocimiento y/o un nuevo modo de acción... (Martínez Llantada, 1996, p. 13). La creatividad ... permite al hombre penetrar en la esencia de los fenómenos estudiados, a utilizar nuevos procedimientos, introducir elementos novedosos en los métodos... (Martínez Llantada, 1996, p. 14).

Como dimensión del contenido, la creatividad le permite al sujeto asimilar un contenido diferente del que se le enseña, como resultado de su papel transformador ante la solución de un problema social.

Los contenidos del proceso docente-educativo en la unidad docente son la sistematización, integración, generalización y aplicación del currículo académico, laboral-productivo, e investigativo y de autopreparación, recibido en la universidad; formación de nuevos conocimientos teóricos, habilidades y valores propios del estadio de desarrollo de la empresa (incluidos contenidos extracurriculares diferenciados, como nuevos métodos, tecnologías, etc.), por medio de la autopreparación.

En la unidad docente, el estudiante insertado se apropia de la cultura de la empresa y de su entorno social, además de sistematizar todos los contenidos asimilados con anterioridad del currículo académico. Estos contenidos incluyen los conocimientos teóricos nuevos que requerirá para buscarle solución al problema profesional real que enfrenta, por medio de la investigación científica; los relativos a la disciplina tecnológica y laboral; los provenientes de la experiencia del personal de la empresa. Por otro, lado se asimilan habilidades propias del perfil de la profesión, entre las cuales están las relacionadas con los medios y las tecnologías pertenecientes a la empresa que le resultan novedosos; las propias de la investigación científica concreta propia del campo de actuación; las necesarias para el trabajo en grupos multidisciplinarios. Además, se establecen los nexos en el desarrollo ascendente de las habilidades, desde las teóricas hasta las profesionales, consolidadas en competencias necesarias para el papel transformador del medio. El postgrado debe ser una continuidad de 
este proceso transformador. Asimismo se adquieren nuevos valores de socialización (relaciones laborales, de trabajo en grupos), de la disciplina del trabajo, de la responsabilidad y el compromiso de la búsqueda de respuestas a los problemas reales de la empresa; esto influye en el desarrollo personal. El trabajo en la unidad docente contribuye al desarrollo de sentimientos, convicciones, actitudes y sensibilidad, que se constituyen también en competencias del profesional que el país necesita en sus graduados universitarios.

\subsection{El método}

Es la secuencia u ordenamiento del proceso docente-educativo; es el componente del proceso que expresa su configuración interna. Refiere cómo se desarrolla el proceso para alcanzar el objetivo, es decir, el camino, la vía que se debe escoger para lograr el objetivo con la mayor eficiencia. La palabra método proviene del griego methodos que literalmente significa "camino hacia algo", su acepción más general es modo de alcanzar el objetivo, actividad ordenada de cierta manera (Diccionario de Filosofía, 1984).

La mayoría de las definiciones de método en la didáctica lo relacionan con la actividad del profesor, integrada o no a la actividad del estudiante, o con la secuencia de pasos o conjunto de procedimientos didácticos que lleven al logro del objetivo.

\subsubsection{Síntesis de las propiedades del método:}

- Siempre existe un objetivo que encausa la actividad consciente del sujeto.

- La actividad del sujeto se conforma como un sistema de acciones.

- Presupone el empleo de medios.

- La acción del método recae sobre un objeto.

- Siempre conduce a un resultado.

(Labarrere y Valdivia, 1988, p. 102; MINED, 1984, p. 249; Klingberg, 1990, p. 267)

\subsubsection{Clasificación de los métodos.}

Los criterios de diversos autores difieren al clasificar los métodos de enseñanza-aprendizaje, así Klingberg (1990, p. 289) los agrupa de acuerdo con el tipo de relación en la actividad profesor-alumno en expositivos, trabajo independiente y de elaboración conjunta. Labarrere y Valdivia (1988) los agrupan de acuerdo con la fuente de adquisición del aprendizaje en orales, trabajo con fuentes impresas e intuitivos. Lerner y Stkatkin usan como criterio el carácter de la actividad cognoscitiva y de acuerdo con ello, los dividen en explicativoilustrativo, reproductivo, de exposición problémica, de búsqueda parcial (heurístico) e 
investigativo. Otros autores los colocan en dos grandes grupos, reproductivos y productivos, incluyéndose dentro de estos últimos el investigativo (Danilov y Stkatkin, 1981; Labarrere y Valdivia, 1988, p. 119; MINED, 1984, p. 256).

En el caso del proceso docente-educativo que se desarrolla en la unidad docente el aprendizaje, de acuerdo con el nivel de asimilación del contenido, es creativo-innovativo; por lo que el método, de acuerdo con este criterio, es investigativo-productivo. Con base en el carácter de la actividad cognoscitiva que desarrolla el estudiante, el método es investigativo, por la relación que se produce entre los sujetos del proceso, es colectivo, de trabajo en grupos, lo que se corresponde con el carácter multidisciplinario de la investigación contemporánea.

El uso del método investigativo en el proceso docente-educativo en la unidad docente, es consecuencia de la aproximación del método fundamental de la ciencia con el carácter de la docencia que se da en la práctica investigativo- laboral, caracterizada por la triada cienciadocencia-producción que se manifiesta en la unidad docente. El método investigativo en la práctica investigativo-laboral en la unidad docente, va dirigido a la solución de un problema real de la empresa, que genera un problema de aprendizaje, de búsqueda del contenido que responde a objetivos curriculares, devenidos objetivos de investigación encaminados a buscar una solución productiva / servicios; por lo que el método en el proceso docenteeducativo en la unidad docente, tendrá las siguientes propiedades:

- Existencia de un problema profesional productivo o de los servicios en la empresa, que es a su vez, un problema de aprendizaje para el estudiante.

- La definición de un objetivo que debe lograr el estudiante (de aprendizaje) en la empresa (productivo o de los servicios).

- La presencia de un objeto (real o virtual, material o ideal) en el que existe el problema y que debe ser transformado, de cumplirse el objetivo.

- El empleo de medios propios de la producción o los servicios, devenidos en medios de aprendizaje.

- La organización de la actividad del estudiante en una secuencia de acciones propias del método de investigación científica.

- Conduce a un resultado de investigación devenido en resultado productivo o de los servicios para la empresa, y de transformación del sujeto.

- Debe singularizar la independencia del trabajo de cada estudiante, dentro de un colectivo que atiende un problema multidisciplinar, e integrar las partes. 


\subsubsection{Métodos problémicos}

La esencia de la utilización de los métodos problémicos en la práctica investigativo-laboral está en la concepción contemporánea de utilizar los métodos de la ciencia en el proceso docente-educativo, lo que se conjuga con esta forma de proceso que tiene sus objetivos en el nivel creativo de asimilación del contenido.

La función básica de los métodos problémicos es la educación del pensamiento creador (Martínez, 1987, p. 94) y de la independencia cognoscitiva, ellos aproximan la enseñanza a la investigación científica (Martínez, 1987, p. 90).

El punto de partida para inducir al estudiante al empleo del método problémico, es el enfrentamiento a una situación problémica. A partir de esta, se plantea el problema, en su trinidad de docente para el profesor, laboral para la empresa, e investigativo para el estudiante; siempre que la situación problémica derive un problema de investigación, lo cual no siempre ocurre (Lerner, 1974, citado por Martínez, 1987, p. 150), a partir del cual se diseña la investigación científica.

El método como activador del proceso docente - educativo en la unidad docente, conjugará lo problémico como esencia de lo investigativo, lo productivo como esencia de lo laboral, lo participativo como esencia de lo interdisciplinar.

La motivación permanente del proceso, debe caracterizar al método. Sin motivación la educación y la instrucción son ineficientes, se hace necesario cohesionar los factores afectivos y motivacionales con el fin de facilitar la comprensión consciente de la necesidad de resolver el problema. La comunicación profesor-estudiante y de estos entre sí es la vía para que estos concienticen la necesidad y formen el motivo.

Es por la vía del método, durante la práctica investigativo - laboral, que el estudiante domina el contenido, convirtiendo lo social (la cultura) en patrimonio individual.

La lógica de la ciencia caracteriza al método en su dimensión instructiva, pero la lógica de la profesión lo caracteriza en su dimensión educativa, lo rector es lo educativo, pues solucionando el problema se resuelve la necesidad social. Por ello denominamos la formación en la unidad docente como investigativo - laboral.

El método es el que va determinando el modo en que debe estructurarse el proceso docente - educativo para garantizar la asimilación del conocimiento y el dominio de la habilidad por el estudiante, que se prepara de ese modo para trabajar, para vivir, una vez egresado, desarrollando su propio método de aprendizaje. 


\subsection{Las relaciones interdisciplinares de los objetivos, contenidos y métodos en el proceso docente- educativo en las unidades docentes}

La división y clasificación en materias o asignaturas con contenidos aislados, agrupados por disciplinas, solo la establece el hombre como una vía para el estudio y análisis a profundidad de las partes constitutivas que integran esa realidad con el compromiso de integrarlas nuevamente para el análisis de los fenómenos en sí, recuperando de esta forma todos los nexos interdisciplinarios, multidisciplinarios y transdisciplinarios de los mismos (Fernández de Alaiza, B. 2000, p. 8). Para Ander-Egg, (1994) la interdisciplinariedad es una forma de preocupación por tender hacia la unidad del saber, habida cuenta de la realidad como totalidad y por ello, las razones y la necesidad de un abordaje interdisciplinar surgen del mismo hecho de asumir la complejidad de lo real. Para Fiallo (2001) la interdisciplinariedad es un proceso y una filosofía de trabajo, es una forma de pensar y de proceder para enfrentar al conocimiento de la complejidad de la realidad y resolver cualquiera de los complejos problemas que esta plantea. Este autor reconoce cuatro paradigmas interdisciplinarios: intradisciplinar, multidisciplinar, interdisciplinar y transdisciplinar. De acuerdo con Salazar (2001, p. 54), se considera que hay coincidencia en señalar que la multidisciplinariedad es el nivel primario de relación interdisciplinaria, que permite lograr la interdisciplinariedad como nivel medio-estructurador y la transdisciplinariedad como el nivel superior. Hay criterios de clasificaciones que identifican otros niveles, aunque en opinión de la autora son gradaciones entre estos tres niveles básicos.

De los diferentes paradigmas de integración interdisciplinar que se manejan hoy día, los más extendidos son el interdisciplinar, el multidisciplinar y el transdisciplinar (López, 1995). Esta clasificación es originalmente de Piaget, pero este autor da unas definiciones que a juicio del autor de este trabajo, son más explícitas.

La interdisciplinariedad se manifiesta, cuando se producen, entre las disciplinas, intercambios mutuos que inspiran métodos, modelos o estructuras comunes y que conforman especies de "híbridos disciplinares". En algunos diseños curriculares de carreras se aplica este modelo, lo que sintetiza el número de disciplinas y pretende la integración entre ellas.

La multidisciplinariedad implica incorporar a la solución de un problema aportaciones procedentes de distintas disciplinas; pero sin que cada una de ellas pierda su individualidad o parcela de competencia definida de antemano. 
La transdisciplinariedad presupone la existencia de significados profundos compartidos por un conjunto de disciplinas, que pueden circular de unas a otras, configurando una red o sistema "omnicomprensivo".

En el proceso docente-educativo que se ejecuta en las unidades docentes, la relación interdisciplinar se da en la unidad dialéctica de interrelación multi-inter y transdisciplinar: Cada paradigma de integración interdisciplinar es portador de los demás, pero en el proceso formativo en lo investigativo-laboral, se prioriza uno $u$ otro en distintas etapas del proceso. Interdisciplinar: Esta noción se manifiesta en el papel de la disciplina principal integradora, especie de "híbrido disciplinar" encargada de atender la formación de modos de actuación profesional, de la aplicación de los métodos que responden a la lógica de la profesión.

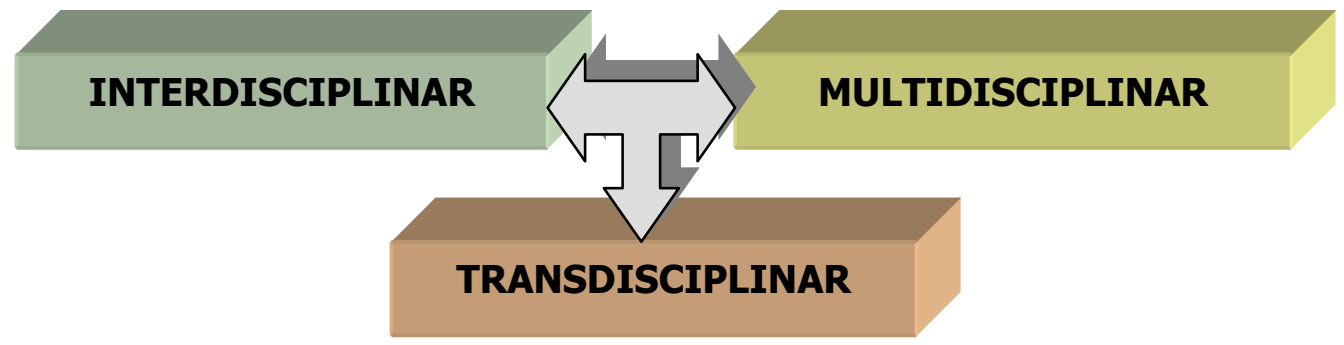

Multidisciplinar: Integrador de asignaturas, disciplinas, años y carreras en la solución de los problemas multivariados y complejos de la empresa. El trabajo científico dirigido a la solución de los problemas que aparecen en la producción y los servicios tienen cada vez más una integración interdisciplinar de este paradigma, porque así son los propios problemas. La especialización creciente de los profesionales hace del trabajo en grupos multidisciplinarios una necesidad de la investigación científica. Los problemas se abordan en su integridad y no en sus aspectos puntuales. Para que el nuevo profesional pueda desarrollar cabalmente sus funciones, debe educarse incorporando hábitos y habilidades que le permitan en su futura vida laboral valorar nuevas ideas, hacer uso adecuado del lenguaje, de la crítica y el debate científico, como vía de desarrollo en la búsqueda de soluciones a los problemas.

Transdisciplinar: Integrador de todas las asignaturas y disciplinas involucradas en la formación de habilidades y valores generalizadores en el estudiante (modos de actuación profesional). Todo educador del claustro de una carrera tiene entre sus objetivos fundamentales, la contribución a la formación profesional del estudiante. El docente debe ser consciente del papel que le corresponde jugar en el proceso y enfrentar el desafío de dar frente a esta problemática, para lo cual debe poseer conocimientos de carácter 
epistemológico, históricos y específicos sobre su disciplina, sobre las ciencias de la educación y sobre la práctica docente.

\subsection{El medio de enseñanza}

Son los objetos con ayuda de los cuales se desarrolla el proceso docente-educativo. Es el componente del proceso docente - educativo que manifiesta el modo en que se expresa el método a través de distintos tipos de objetos materiales. En la definición misma de medio de enseñanza se hace evidente que éste es el vehículo, mediante el cual, se manifiesta el método, o sea, que es el portador material del método. El medio principal del proceso docente-educativo en la unidad docente, es la empresa donde se inserta la propia unidad docente, concebida como el soporte material del proceso.

La importancia del empleo de los medios en el proceso docente - educativo fue destacada por Comenio (1592 - 1670) desde los albores de la didáctica "para aprender todo con mayor facilidad deben utilizarse cuantos más sentidos se puedan;... deben ir juntos el oído con la vista y la lengua con la mano... (Comenio, 1983, p. 124); ...será bueno que todo lo que se acostumbra a tratar en clase, esté pintado en las paredes del aula" (Comenio, 1983, p. 125).

Comenio llega a incluir, en las once reglas del método de las artes, la regla III, que indica "debe enseñarse el empleo de las herramientas con ellas mismas, no con palabras; esto es, con ejemplos mejor que con preceptos" (Comenio, 1983, p. 171).

En la discusión de los principios didácticos puede verse que la mayoría de los autores coinciden en incluir dos (unidad teoría - práctica y unidad concreto - abstracto o de visualización) que tienen a los medios de enseñanza en el centro del proceso. Este carácter meramente visualizador de los medios es criticado por González (1990), cuando expresa que "reducir el papel de los medios de enseñanza al enfrentamiento del estudiante con lo concreto, es propio de una posición sensualista del proceso del conocimiento y limita sus posibilidades reales" (González, 1990, p. 49). De esta forma, se reduce el proceso del conocimiento a la formación del conocimiento empírico. La conceptualización de los medios de enseñanza se ha ampliado en la medida que avanza la tecnología y hoy hay cada vez criterios más amplios sobre los mismos. Galkan (1973) los denomina como aquellos medios materiales necesitados por el profesor y los estudiantes para una estructuración y conducción efectiva y racional del proceso de instrucción y educación a todos los niveles, en todas las esferas del sistema educacional y para todas las asignaturas, para satisfacer las exigencias del plan de enseñanza. González (1990, p. 48) define el medio de enseñanza en sentido restringido, circunscrito al proceso docente - educativo, como todos los componentes 
del proceso que actúan como soporte material de los métodos con el propósito de alcanzar los objetivos. Un criterio amplio de medio enseñanza abarca a todo aquello que contribuye a la efectividad del proceso y no lo restringe a ciertos objetos materiales (García Galló, 1983, p. 149). Para más precisión aquí la efectividad debe entenderse como calidad e incluir la eficiencia y eficacia del proceso.

En la definición más general y contemporánea del medio de enseñanza, se alude al mismo como el que se desarrolla el proceso docente-educativo, pero muchas clasificaciones de autores incluyen entre ellos el dónde se desarrolla el proceso. Así el aula, los laboratorios docentes, las parcelas de experimentación, talleres, lugares de excursiones escolares, etc.; se incluyen entre estos. Klingberg (1990) los denomina "generales"; Dale "de experiencias artificiales o directas"; de experiencias directas con la realidad (Llerena, 1983); de cuarta generación (Schramn, citado por González, 1990, p. 76); o de entrenamiento según Jamov (1971).

Las diferentes clasificaciones de los medios de enseñanza responden a la forma clásica del proceso docente - educativo denominada clase y en muchas de ellas se refieren a clasificaciones de objetos aislados o conjuntos de ellos con funciones didácticas específicas. Solo en la clasificación de Edgar Dale (citado por González, 1990, p. 77), denominada "el cono de la experiencia", aparecen en la base del cono las "experiencias directas con propósito" como el medio de más nivel de concreción.

La empresa donde se inserta la unidad docente es el sistema de medios que permite el desarrollo del proceso docente - educativo para el desarrollo de la práctica investigativolaboral, por ello a continuación se estructura el modelo de empresa donde se inserta una unidad docente modelo, que responda a las regularidades del modelo didáctico.

Esta unidad docente poseerá características propias:

- La empresa debe ser rentable y eficiente.

- Tener habilitados locales para el albergue de los estudiantes, en el caso de que sea aconsejable que los estudiantes permanezcan en él y para impartir docencia, tanto de pregrado a los estudiantes (fundamentalmente de contenidos extracurriculares), como de postgrado a los trabajadores de la empresa, del territorio o la comunidad.

- Disponer de los medios de enseñanza audiovisuales e informáticos necesarios para el desarrollo de la labor docente e investigativa en sus propias instalaciones y tener las condiciones materiales óptimas, en su base de equipamiento tecnológico, para dar respuesta a sus necesidades de producción o servicios, y la disposición de ponerlas en manos de los estudiantes de práctica. Garantizar las condiciones materiales para el 
desarrollo de la práctica investigativo- laboral de los estudiantes (transporte, laboratorios, talleres, etc.)

- Tener una biblioteca de uso común para los trabajadores y estudiantes insertados y que combine una bibliografía y hemerografía científico - técnica actualizada, con literatura general.

- Tener profesionales de la producción, categorizados como profesores adjuntos de la universidad y los recursos humanos capacitados necesarios para atender la práctica investigativo- laboral de los estudiantes.

- Contar con un banco de problemas, abierto a los estudiantes insertados, para su solución, y generar problemas de la producción o los servicios, cuya solución requiera del trabajo multidisciplinario de los estudiantes, o que puedan asimilar a estudiantes de varias carreras universitarias.

- Abrir sus fuentes de información, al uso por los estudiantes en función de la solución de los problemas de la empresa, cuidando las medidas de seguridad informática y compartimentación más pertinentes y brindar acceso a los estudiantes a todas las áreas de producción y servicios de la empresa, donde se generan los problemas de la misma.

- Priorizar la participación de la universidad en los convenios de investigación y desarrollo, y prestación de servicios científico técnicos, para la solución de los problemas de la empresa.

- Mantener actualizado el diagnóstico de las necesidades de superación del personal de la empresa, sobre la base del cual elabore un plan de superación en convenio con la universidad (Debe incluir la superación pedagógica de los profesores adjuntos y una estrategia de grados académicos y científicos: especialistas, máster y doctores).

- Tener áreas deportivas y para actividades culturales y recreativas previstas entre la empresa, la comunidad y la universidad.

- Priorizar la introducción de los resultados científicos técnicos de los estudiantes en la solución de los problemas de la empresa. Celebrar encuentros de generalización periódicos con la universidad.

- Tener elaborada una estrategia para la recepción de recién graduados de la universidad, en los periodos de adiestramiento laboral.

- Disponer de un marco financiero que le permita apoyar a la universidad en los procesos de docencia - investigación - extensión - gestión, que deben realizar los estudiantes, considerando esto como una inversión para el futuro y no como un gasto de la empresa. 
La universidad necesita buscar sus unidades docentes que respondan a una nueva concepción de empresas generadoras de problemas (indicador de su desarrollo), cuyas soluciones sean necesidades reales y abordarlos en su carácter multidisciplinario, por grupos de estudiantes con igual carácter, que por medio de su tarea individual, contribuyan a la solución colectiva del problema. La empresa en la que se inserta la unidad docente, como medio del proceso docente - educativo para la formación investigativo- laboral de sus estudiantes, debe concebirse como medio de la universidad y no de un área organizativa de esta. Investigar cuáles estudiantes insertan cada curso en ella, depende del carácter de los problemas reales a los que busca solución. Este es el concepto que se defiende.

\subsection{La forma de enseñanza}

Son los aspectos organizativos más externos. El proceso docente - educativo se organiza en el tiempo, en correspondencia con el contenido a asimilar y el objetivo a alcanzar; así mismo, se establece una determinada relación entre los estudiantes y el profesor, en esto coinciden la mayoría de los autores

Las formas organizativas constituyen el componente integrador del proceso de enseñanza - aprendizaje, esto se evidencia en la manera en que se ponen en interrelación todos los componentes personales y no personales del proceso. Las formas reflejan las relaciones entre profesor y estudiantes en la dimensión espacial y temporal del proceso. Responden a la pregunta: ¿Cómo organizar el proceso docente - educativo?... (González, Recarey, Addine, en Addine y otros, 2002, p.79)

Forma de organización de la enseñanza es: la manera en que se manifiesta externamente la relación profesor - alumno, es decir, la confrontación del alumno con la materia de enseñanza bajo la dirección del profesor. (Labarrere y Valdivia, 1989, p. 138.)

La forma es la organización, el orden que adopta el proceso para alcanzar el objetivo, en el que se destaca primeramente, la relación profeso -estudiante. (Álvarez de Zayas, 1998, p. 5)

Integrando estos conceptos al tipo de proceso que ocupa este trabajo, puede definirse que: La forma del proceso docente - educativo en la unidad docente es la práctica investigativo laboral, que no debe confundirse con la práctica laboral como tipo de clase, comparable con la práctica de laboratorio, de campo, etc.

Esta tiene sus particularidades, que la diferencian de la forma denominada clase, y que aparece definida en este trabajo. 


\subsection{La evaluación}

Es el componente que expresa las transformaciones que se lograron alcanzar en el estudiante, es el producto que se obtiene del proceso. Como resultado es un componente del proceso docente-educativo que se manifiesta por el estado final alcanzado en dicho proceso, que satisface o no el objetivo programado.

La evaluación del proceso docente-educativo en la unidad docente estará acorde con la calidad del resultado en la solución del problema, planteado al estudiante o grupo de ellos.

En cada período de vinculación de estudiantes en la empresa, debe hacerse una evaluación de todos los resultados de la relación universidad - empresa, por tener una incidencia totalizadora en las transformaciones experimentadas por los estudiantes insertados. Los beneficios que la unidad docente ha de aportar, tanto a la universidad como a la empresa hacen que en la evaluación de los resultados de estas deban medirse los cambios en los indicadores productivos de las áreas a las que se vincula, lo que significa que la universidad asume un compromiso con estos resultados y, por tanto, en la valoración de su gestión debe considerarse su grado de cumplimiento.

En los resultados de la unidad docente debe de medir la calidad con la que se forman los profesionales en la misma, así como el desarrollo y resultados de los proyectos de investigación que en ella se realizan y del nivel de satisfacción que se logra en la cobertura de las necesidades de superación de los profesionales, lo que significa que la empresa asume un compromiso con estos resultados y, por lo tanto, en la valoración de su gestión ha de considerarse el grado de cumplimiento de los mismos. Es decir, que en la evaluación institucional de la misma deben estar presentes los resultados que, en la formación de los profesionales, las investigaciones y la superación se alcancen en la unidad docente.

\subsection{Procesos universitarios}

La docencia es un proceso que articula el desarrollo de conocimientos, habilidades, valores, capacidades y formación trascendente de la personalidad (convicciones, sentimientos, voluntad, actitudes) presentes en el proceso docente-educativo, como un proceso consciente que se articula en la relación del que enseña - el que aprende y desarrolla la creatividad de los mismos. Su gestión se hace visible en los planes de estudio y los currículos. Estos deben garantizar, en el proceso, la formación integral a partir de las dimensiones instructiva, educativa y desarrolladora, el cual debe ser eficiente y eficaz.

La investigación: Este proceso tiene como funcione, la innovación y el desarrollo del conocimiento científico, tecnológico y social. En esta célula organizativa todos los procesos 
son problémicos. La investigación objetiva el quehacer de la educación superior en cuanto a producción de conocimiento para el desarrollo social y humano.

La Extensión: Proceso de promoción de la cultura, hacia el contexto social. Está presente en la vida de los comunidades y sociedades, y deben tender a resolver situaciones y necesidades sentidas por los diferentes sectores de la sociedad.

La Gestión: Es el hilo conductor y facilitador de la realización de los procesos y actividades, el cual tiene un desarrollo en sí mismos y posibilita la acción con los demás. Actividad de gobierno y administración que hace referencia cómo es el proceso de adopción de decisiones.

\section{CONCLUSIONES}

En apretada síntesis, el trabajo ha pretendido presentar los elementos esenciales de una propuesta de didáctica que sistematice un tipo de proceso docente-educativo muy particular que se desarrolla en las empresas, devenidas en unidades docentes de las universidades, durante el desarrollo de las prácticas denominadas investigativo-laborales.

La propuesta didáctica se fundamenta en las dos relaciones dialécticas esenciales que se han definido para este proceso, las de estudio-trabajo y la de ciencia-tecnología; así como en las ocho regularidades encontradas en el mismo.

La didáctica para el proceso docente-educativo en las unidades docentes reúne las más actualizadas concepciones epistemológicas de esta ciencia y responde a la necesidad de fundamentar, teóricamente, un tipo de proceso didáctico que posee sus particularidades propias y, con ello, sobrepasar el nivel esencialmente empírico actual con que se desarrolla. La unidad docente modelada se constituye en el sistema de medios de enseñanza que propician la materialización del proceso docente - educativo que se propone para el desarrollo de la práctica investigativo - laboral en las empresas vinculadas a la universidad. La empresa en la que se inserta la unidad docente, concebida como medio del proceso docente - educativo que en ella se desarrolla, debe ser integradora de los paradigmas de relación interdisciplinar incluidos en el modelo, para ello debe generar problemas multivariados y estar abierta a la formación del profesional. Como complementación del análisis didáctico presentado, se elaboró el modelo para la relación universidad - empresa que responda a las necesidades derivadas de la propuesta didáctica presentada, el cual no se ha incluido en el trabajo.

El proceso docente - educativo en las unidades docentes tiene sus especificidades, pero su fundamento teórico pedagógico y metodológico está contenido en la Pedagogía y la 
Didáctica que estudian los procesos formativos y específicamente los de carácter docenteeducativos. Las especificidades de este proceso han llevado a la modelación de su propia didáctica.

\section{REFERENCIAS}

Addine, F. (1997). Didáctica y currículo. Análisis de una experiencia. Potosí: Ed AB.

Addine F. y otros. (2002). Didáctica, teoría y práctica. La Habana: ISP "E, J. Varona”.

Álvarez de Zayas, C. (1998). Fundamentos teóricos de la dirección del proceso docente educativo en la Educación Superior Cubana. La Habana: MES.

(1990). Fundamentos teóricos de la dirección del proceso docente educativo en la educación superior cubana. La Habana: Imprenta "Andrés Voisin" ENPES.

Varela-MERCADU S.A.

(1992). La Escuela en la Vida. La Habana: Editorial Félix . (1995). La Pedagogía Universitaria. Una experiencia cubana. Ponencia en Pedagogía '95, La Habana.

Pueblo y Educación.

(1999). La Escuela en la vida. (3ra Edición). La Habana: Editorial

Álvarez de Zayas, C. y González, E.U. (1998). La Didáctica: un proceso consciente de enseñanza y aprendizaje. Cintex (7): 1998, 5 - 9.

Álvarez de Zayas, R. M. (1997). Hacia un currículum integral y contextualizado. La Habana: Editorial Academia.

Babanski, Yu. K. (1982). Optimización del proceso de enseñanza. La Habana: Editorial Pueblo y Educación.

Castellanos, D. y otros. (2001). Hacia una concepción del aprendizaje desarrollador. La Habana: Colección Proyectos.

Comenio, J. A.(1983). Didáctica Magna. La Habana: Pueblo y Educación.

Danilov, M. A. y Stkatkin, M. N. (1978). Didáctica de la escuela media. La Habana: Editorial de Libros para la Educación.

Editorial de Libros para la Educación.

(1981). Didáctica de la escuela media. La Habana:

Diccionario de filosofía. (1984). Moscú: Editorial Progreso. 
Fernández, B. (2000). La interdisciplinariedad como base de una estrategia para el perfeccionamiento del diseño curricular de una carrera de ciencias técnicas y su aplicación a la Ingeniería en Automática en la República de Cuba. Tesis de doctorado en Ciencias Pedagógicas, Instituto Superior Politécnico "José A. Echevarría", La Habana, Cuba.

Fiallo, J. P. (2001). La interdisciplinariedad en la escuela: de la utopía a la realidad. La Habana: Instituto Central de Ciencias Pedagógicas, (Formato electrónico. 16 p).

Fuentes, H. (2000). Didáctica de la Educación Superior. Bogotá: Escuela Superior Profesoral, INPAHU.

Fuentes, H. y Álvarez, I. (1998). Dinámica del proceso docente educativo en la educación superior. Santiago de Cuba: CEES Universidad de Oriente.

Galkan, D. (1973). Acerca de la importancia de los medios técnicos de enseñanza. Ponencia en conferencia de expertos de países socialistas, Moscú.

García Galló, G. J. (1983). Los medios de enseñanza a la luz de la dialéctica materialista, Revista Varona, (11), 147 - 151.

González, F. (1993). Problemas Epistemológicos de la Psicología. México: UNAM.

González, P. O. y col. (1995). Didáctica Universitaria. La Habana: CEPES.

González, V. (1990). Teoría y práctica de los medios de enseñanza. La Habana: Editorial Pueblo y Educación.

Herrera, J. L. (2003). Un modelo del proceso docente - educativo en las unidades docentes para el desarrollo de la práctica investigativo - laboral. Tesis doctoral en Ciencias Pedagógicas, Universidad de Pinar del Río, Cuba.

Jamov, P. F. (1971). Los medios técnicos de instrucción y su empleo en el proceso de enseñanza. Tesis doctoral en Pedagogía, Instituto Lenin, Moscú.

Klingberg, L. (1990). Introducción a la Didáctica General. La Habana: Editorial Pueblo y Educación.

Labarrere, G. y G. Valdivia. (1989). Pedagogía. La Habana: Editorial Pueblo y Educación.

Lazo, J. (1996). La universidad al servicio de la sociedad. Sucre: Universidad Andina.

López, F. (1995). Una nueva fuente de inspiración para la educación científica, Revista Enseñanza de las Ciencias, (13, 2), 249-256.

Llerena, J. M. (1983). Estudio comparativo de las clasificaciones de los medios de enseñanza, Tesis de la Facultad de Superación del ISP Varona, La Habana.

Martínez, M. (1987). La enseñanza problémica de la filosofía marxista - leninista. La Habana: Editorial de Ciencias Políticas. 
(1996). Calidad educacional, actividad pedagógica y creatividad. La Habana: Editorial Academia.

MINED. (1984). Pedagogía. La Habana: Editorial Pueblo y Educación.

Salazar D. (2001). La formación interdisciplinaria del futuro profesor de Biología en la actividad científico - investigativa, Tesis de doctorado en Ciencias Pedagógicas, I. S. Pedagógico "E. J. Varona", La Habana, Cuba.

(2001). Hacia la construcción de una Didáctica Interdisciplinaria. La Habana: Facultad de Humanidades, ISPEJV, (Formato electrónico).

Silvestre, M y J. Zilberstein. (2000). Reflexiones acerca de la necesidad de establecer Principios Didácticos, para un proceso de enseñanza aprendizaje desarrollador, en Enseñanza y Aprendizaje Desarrollador. México: Ediciones CEIDE, (2 - 18).

Skatkin, M. N. (1979). Didáctica: perfeccionamiento del proceso de la enseñanza. La Habana: Editorial Pueblo y Educación.

Tomaschewskl, K. (1966). Didáctica General. México: Editorial Grijalbo S.A.

Vargas, A. (s.f.). Didáctica. La Habana: CEPES Universidad de la Habana, (Formato electrónico, 65 p). 